in nonhuman primates. At a single neuron level, primate $\mathrm{V} 1$ responses show signals that change according to the distance of an object $^{13,14}$, forming a potential neural substrate for the dynamic changes observed at a much coarser spatial scale by Murray and colleagues.

Indeed, at a fine-grained level, their findings also raise intriguing questions about whether a V1 representation of the environment that reflects perceived depth and size can be internally coherent. For example, when two objects at different perceived depths partially occlude each other, correct border assignments may be particularly complex as portions of the objects adjacent to the border may be relatively displaced according to perceived depth. The current observation that the near and far objects were judged to appear both smaller and larger with respect to an equivalently sized two-dimensional object may suggest a 'push-pull' mechanism for maintaining coherence in a spatially distributed V1 representation of the subjects perceptions.

Taken together, these compelling findings force us once again to consider a revised model of visual processing in which $\mathrm{V} 1$, far from being a passive feed-forward recipient of retinal signals, instead flexibly combines retinal and extraretinal signals to potentially build an integrated representation of the perceived visual environment. Future study of how V1 activity relates to human consciousness will doubtless continue to be both interesting and informative.
1. Emmert, E. Klin. Mbl. Augenheilk. 19, 443-450 (1881)

2. Murray, S.O., Boyaci, H. \& Kersten, D. Nat. Neurosci. 9, 429-434 (2006)

3. Haynes, J.D., Lotto, R.B. \& Rees, G. Proc. NatI Acad. Sci. USA 101, 4286-4291 (2004).

4. Shikata, E. et al. J. Neurophysiol. 85, 1309-1314 (2001).

5. Tsutsui, K., Sakata, H., Naganuma, T. \& Taira, M Science 298, 409-412 (2002).

6. Weiskrantz, L., Cowey, A. \& Hodinott-Hill, I. Nat. Neurosci. 5, 101-102 (2002)

7. Tong, F. Nat. Rev. Neurosci. 4, 219-229 (2003).

8. Hupe, J.M. et al. Nature 394, 784-787 (1998).

9. Glickstein, M. \& Whitteridge, D. Trends Neurosci. 10, 350-353 (1987)

10. Holmes, G. Br. J. Ophthalmol. 2, 353-384 (1918).

11. Anderson D.H. \& van Essen D.C. Proc. Natl. Acad. Sci. USA 84, 6297-6301 (1987)

12. Whitney, D. et al. Science 302, 878-881 (2003).

13. Dobbins, A.C., Jeo, R.M., Fiser, J. \& Allman, J.M. Science 281, 552-555 (1998).

14. Trotter, Y., Celebrini, S., Stricanne, B., Thorpe, S. \& Imbert, M. Science 257, 1279-1281 (1992).

\title{
Quantifying motor neuron loss in ALS
}

Amyotrophic lateral sclerosis (ALS or Lou Gehrig's disease) leads to paralysis from the death of motor neurons in the spinal cord and brainstem. It is incurable, and patients typically die within three to five years of disease onset. Neurodegenerative diseases like ALS can progress slowly, with years of clinically undetectable symptoms followed by rapid deterioration. Although ALS selectively targets motor neurons, it has remained unclear whether particular synapses are selectively targeted and whether these synapses are lost gradually or abruptly.

In an article in this issue (page 408), Pico Caroni and colleagues addressed this issue by creating a quantitative map of the innervation of hindlimb muscle compartments by motor neurons in the mouse. They then went on to study the mechanisms of early disease progression in a mouse model of ALS.

Motor neurons innervating skeletal muscle fibers are subdivided into three functional subtypes - fast twitch and fast fatiguable (FF), fast twitch and fatigue resistant (FR) and slow twitch (S). The authors used transgenic mice expressing green fluorescent protein in only a few neurons and mapped the distribution of all synapses made by individual motor neurons in the lateral gastrocnemius muscle. Once they created a topographic map of motor neuron innervation, they analyzed

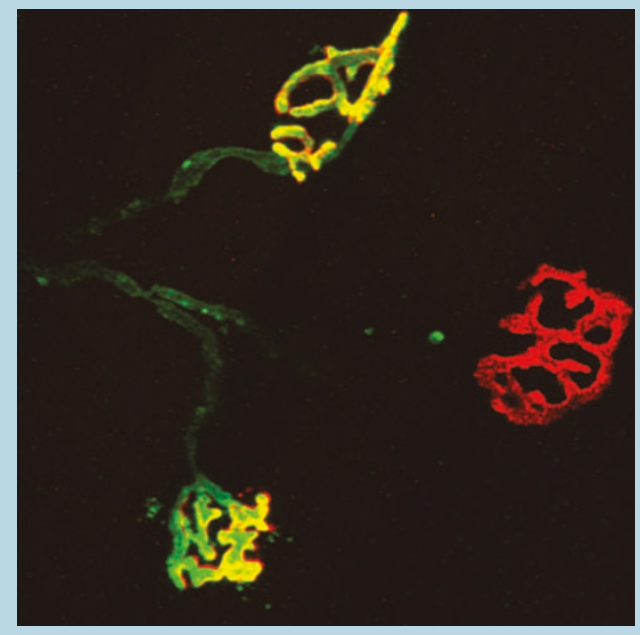
denervation patterns in mice containing a mutation in the enzyme superoxide dismutase (SOD1). In this familial ALS mouse model, they found that FF axons were selectively affected early on in the disease and that these abruptly disconnected from their peripheral synapses when the mice were 48-52 days old. FR motor neurons innervating the same muscle compartments compensated initially for this loss by reinnervating neuromuscular junctions (NMJs) on the muscle fibers, but over time, were less able to maintain the additional NMJs. They then started pruning their nerve branches by the time the mice were 80-90 days old. S-type motor neurons were particularly resistant to disease, and maintained expanded motor units up to the time the mice died.

What makes the FF and FR motor neuron axons selectively vulnerable to disease? The authors did cross-innervation experiments and nerve crush studies and concluded that the early vulnerability of FF motor neurons reflects a vulnerability of the presynaptic motor neuron axon rather than its target muscle or peripheral synapses. They also found that axonal transport was particularly vulnerable in FF and to a lesser degree in FR axons, leading to synaptic vesicle stalling and loss from NMJs. In the figure, the NMJ in the center has lost all synaptic signal (green, synaptic vesicle marker SV2; red, acetylcholine receptor) but is still innervated. Other NMJs may be less affected, like the lower one in the figure. Daily applications of the growth factor ciliary neurotrophic factor (CNTF) protected FF axons from synaptic vesicle loss and peripheral pruning and also helped to maintain the expanded size and innervation of compensating FR motor neuron axons. CNTF helped to boost axonal resistance to disease by causing neurofilament density reductions in both FF and FR neurons and by preventing the upregulation of an anti-apoptotic protein, Bc12a1-a, in motor neurons.

By providing a quantitative account of the selective vulnerability of different motor neuron populations during the progression of disease, this work opens up new possibilities for treating ALS and related motor neuron disorders.

\section{Kalyani Narasimhan}

